\title{
Carbon dioxide baited trap catches do not correlate with human landing collections of Anopheles aquasalis in Suriname
}

\author{
Hélène Hiwat ${ }^{+}$, Rob Andriessen, Marjolein de Rijk, \\ Constantianus Johanna Maria Koenraadt, Willem Takken
}

Laboratory of Entomology, Wageningen University and Research Center, Wageningen, The Netherlands

\begin{abstract}
Three types of carbon dioxide-baited traps, i.e., the Centers for Disease Control Miniature Light Trap without light, the BioGents (BG) Sentinel Mosquito Trap (BG-Sentinel) and the Mosquito Magnet ${ }^{\circledR}$ Liberty Plus were compared with human landing collections in their efficiency in collecting Anopheles (Nyssorhynchus) aquasalis mosquitoes. Of 13,549 total mosquitoes collected, 1,019 (7.52\%) were An. aquasalis. Large numbers of Culex spp were also collected, in particular with the (BG-Sentinel). The majority of An. aquasalis $(83.8 \%)$ were collected by the human landing collection (HLC). None of the trap catches correlated with HLC in the number of An. aquasalis captured over time. The high efficiency of the HLC method indicates that this malaria vector was anthropophilic at this site, especially as carbon dioxide was insufficiently attractive as stand-alone bait. Traps using carbon dioxide in combination with human odorants may provide better results.
\end{abstract}

Key words: Anopheles aquasalis - BG-Sentinel trap - CDC light trap - Mosquito Magnet Liberty Plus - carbon dioxide

Anopheles (Nyssorhynchus) aquasalis Curry 1932 is a neotropical coastal mosquito. It is an important malaria vector in Brazil (Deane 1986, Flores-Mendoza \& Lourenço-de-Oliveira 1996, Póvoa et al. 2003) and a proven or potential vector in neighbouring countries (Rozendaal 1987, Laubach et al. 2001). The species shows diverse biting behaviour throughout its geographic range, with local anthropophilic and endophilic biting patterns (Deane et al. 1948, Ferreira 1964, FloresMendoza et al. 1996). Although its role as malaria vector in the Guianas remains unclear, malaria-infected specimens have been found in Suriname's neighbouring country Guyana (Laubach et al. 2001).

An. aquasalis is a common mosquito in Suriname, often found in high densities in coastal areas. Suriname is divided into two main areas, the partly populated and cultivated coastal area, which entered its maintenance phase in 1968, and a sparsely populated interior covered with primary rainforest, where malaria transmission still occurs. These two areas are separated by a white sand formation called the "Savannah Belt". In the interior, Anopheles darlingi Root is the main malaria vector. Along the coast, An. aquasalis was a suspected malarial vector before 1968. Although its vector role was never proven, in many coastal malaria outbreaks, such as in Moengo $(1946,1977)$ and in the village of Galibi (1972) (Panday 1977, Rozendaal 1990), An. aquasalis was one of the most common anophelines found.

Financial support: WUR, MM-MP, PAHO-Suriname (AMI-Ravedra funds), BOG

+ Corresponding author: helenehiwat@gmail.com

Received 10 December 2010

Accepted 24 March 2011
An. aquasalis can be sampled using human landing collection (HLC); however, this method leaves the collector exposed to potentially malaria-infected mosquitoes and is therefore becoming less accepted on ethical grounds. In addition, collecting An. aquasalis utilising HLC in an environment with high densities of other biting mosquito species, as is often the case in Suriname, is very uncomfortable for the collector. Many alternative methods for collecting mosquitoes have been evaluated to find a suitable alternative to the HLC (Rubio-Palis 1996, Mboera et al. 2000, Laubach et al. 2001, Kline 2002). However, a limited number of these studies were conducted using An. aquasalis as a target species. Light traps are promising candidates to replace HLC in epidemiological monitoring of malaria vectors, especially when used in combination with human bait (Mbogo et al. 1993, Davis et al. 1995, Costantini et al. 1998b). However, the use of light traps for capturing disease vectors is limited because they do not represent the true human-biting mosquito population, reducing their reliability for the estimation of the entomological inoculation rate (Mboera 2005).

Another alternative to HLC are carbon dioxide-baited traps (Mboera \& Takken 1997). Carbon dioxide has long been acknowledged as a mosquito attractant (Van Thiel et al. 1939, Reeves 1953) and this attraction was confirmed for several tropical anopheline species such as An. aquasalis (Gillies 1980, Service 1993, Costantini et al. 1996, Rubio-Palis 1996, Mboera et al. 2000). For instance, Healy and Copland (1995) found that a carbon dioxide concentration level of $0.01 \%$ above background activated Anopheles gambiae females. In general, mosquitoes are collected in greater numbers with increasing levels of carbon dioxide (either from cylinders or from dry ice), until a carbon dioxide level of approximately $500 \mathrm{~mL} / \mathrm{min}$. Typically, no further increase in captures is found above this level (Kline et al. 1991, Mboera \& Takken 1997). 
The goal of this study was to evaluate the efficiency of different trapping devices baited only with carbon dioxide in collecting An. aquasalis mosquitoes and to assess the potency of these traps to replace HLC for entomological surveys or long-term monitoring. Three different traps were compared with the HLC method: the Centers for Disease Control Miniature Light Trap without light (CDC trap), at a level of $1 \mathrm{~m}$ above the ground, the BioGents (BG) Sentinel Mosquito Trap (BG-Sentinel), at ground level, and the Mosquito Magnet ${ }^{\circledR}$ Liberty Plus (MM-Plus), also at ground level.

\section{MATERIALS AND METHODS}

Study design - Between May 5-May 22 2009, three carbon dioxide-baited mosquito traps were tested for their efficiency in collecting An. aquasalis mosquitoes; results were compared with the conventional HLC method. The collections took place along a roadside in a residential area in northern Paramaribo, close to the Atlantic coast. Nearby ecosystems consisted mostly of coastal marshes with abundant mangrove forests. In northern Paramaribo, An. aquasalis mosquitoes bite predominately between 19:00-19:30 h. Before and after this peak, almost no An. aquasalis can be found. Therefore, we collected mosquitoes between 18:45-19:45 h.

This study involved attracting mosquitoes using carbon dioxide as odour bait. Because carbon dioxide is considered a middle to long-range factor in host orientation, it attracts mosquitoes from approximately $15-30 \mathrm{~m}$ away (Gillies \& Wilkes 1969). Therefore, collections were performed at four locations that were separated by at least $50 \mathrm{~m}$ to prevent interference. Traps were rotated over these four locations according to a rotation scheme using a 4 x 4 Latin square design. Over an 18-day period, this rotation scheme was repeated three times, separated by a three-day time interval. Catches were placed in sampling tubes and stored overnight. Temperature and humidity levels were measured during the collecting times. The day immediately following each collection, mosquitoes were sorted and counted per collection method. Anopheles mosquitoes were sexed and identified to the species level; Culex spp and Aedes spp mosquitoes were also collected and identified to the genus level. Three trap models were used, each baited with carbon dioxide.

MM-Plus - In this trap, the MM-Plus converts propane into carbon dioxide (flow $500 \mathrm{cc} / \mathrm{min}$ ), which is used as bait (Kline et al. 2006). The MM-Plus is generally provided with 1-octen-3-ol (octenol) as bait, but in our experiments this compound was not used.

CDC trap - For collection, the CDC trap was placed at approximately $1 \mathrm{~m}$ above the ground. Because of the large number of insects of no importance attracted by the light of this trap, the light was removed and, as with the MM-Plus, only carbon dioxide was used as bait. The carbon dioxide was obtained from a gas cylinder and fed into the trap entrance by plastic tubing. Using a pressure regulator (Concoa, Virginia Beach, Virginia, USA; model CGA 320) and a flow meter (Brooks Instrument, Hatfield, Pennsylvania, USA; model 1355), a constant flow of approximately $500 \mathrm{cc} / \mathrm{min}$ of carbon dioxide (same as MM-Plus) was obtained. The carbon dioxide was released through a tube near the trap opening.

BG-Sentinel - The BG-Sentinel trap was baited with a flow of carbon dioxide of approximately $500 \mathrm{cc} / \mathrm{min}$; carbon dioxide was obtained and released as described for the CDC trap. BG-Lure, a mosquito attractant generally provided with these traps, was not used.

The trap collections were compared with the HLC method. The HLC were performed by four collectors; males and females of average body weight and height worked during the study days in alternating shifts of two persons. HLC were carried out outdoors during the exact same time as when the traps were operative. All collectors sat down on a stool and exposed their lower legs to attract mosquitoes. The amount of skin exposed allowed for a maximum collection effort by the collectors as a result of high mosquito densities. From the resulting number of mosquitoes, a mean number of mosquitoes collected per person, per night was calculated and compared with nightly trap collections. Adjustments were made to the mean and total number of mosquitoes collected per trap to account for missing data.

Statistical analysis - An. aquasalis numbers were $\mathrm{Ln}(\mathrm{x}+1)$ transformed and imported into a General Linear Model (SPSS version 17) to identify differences in total collected An. aquasalis mosquitoes between collection methods. Tukey's HSD (post-hoc) tests were used to find differences between and within periods per collection method. Temperature and relative humidity were taken into account as explanatory factors for differences in mosquito collections. Pearson's correlations were calculated to define possible dependence of the alternative methods in relation to HLC in the number of mosquitoes collected over time.

Ethical considerations - The use of HLC for this study was approved by the ethical committee of the Ministry of Health (MoH) (project VG2006-006, MoH letter reference 406, August 2006). The four collectors were two researchers and two entomology technicians from the Bureau of Public Health of MoH. All collections were done in a coastal area that is free of malaria and that entered its maintenance phase in 1968.

\section{RESULTS}

A total of 13,549 mosquitoes were collected; of these, $7.52 \%$ were An. aquasalis, $91.11 \%$ were Culex spp (the coastal Culex population consists predominately of Culex quinquefasciatus Say) (H Hiwat, unpublished observations) and $0.03 \%$ were Aedes spp. All mosquitoes were females. Because of operational failures, MM-Plus catches were not performed on nights 3, 6 and 11; BG-Sentinel catches were not carried out on nights 4 and 10 . Because between periods of collection no significant differences in total number of An. aquasalis mosquitoes collected were found per sampling method $(p>0.05)$, the results for all periods per collection method were summed.

When adjusted for missing data, overall, the BG-Sentinel collected $52.7 \%$ of all mosquitoes, the CDC light trap $26.7 \%$, the MM-Plus $7.4 \%$ and the HLC (per person) 
$13.2 \%$. Of the An. aquasalis mosquitoes, the BG-Sentinel collected 5.9\%, the CDC light trap 6.4\%, the MM-Plus $15.5 \%$ and the HLC (per person) $72.1 \%$. The overall HLC percentage of An. aquasalis, not corrected with a meanper-person calculation, was $83.8 \%$. The mean number of collected mosquitoes per collection method, per day is shown in Table. The HLC method collected many more An. aquasalis per day compared with any of the other methods $(\mathrm{p}<0.05)$. Both the CDC trap and the BG-Sentinel trap collected large numbers of Culex mosquitoes.

Significant differences in the number of An. aquasalis mosquitoes between the HLC and each of the three other collection methods were found $(p<0.001)$. Between the BG-Sentinel, the CDC trap and the MM-Plus, no significant differences in the mean or total number of collected An. aquasalis mosquitoes were found (for all combinations, $p>0.05$ ). The total number of mosquitoes, the number of An. aquasalis and the number of Culex spp mosquitoes per collecting method per day is depicted in Figure.

Temperature, relative humidity and location did not influence the obtained results $(\mathrm{p}>0.05)$. The mean number of An. aquasalis mosquitoes collected per day with the BGSentinel, the CDC light trap as well as with the MM-Plus was not correlated with the total An. aquasalis mosquitoes collected per day using the HLC method. The Pearson's correlation coefficient values were as follows: BG-Sentinel, 0.27, MM-Plus, 0.30 and CDC trap, 0.64 (Figure).

\section{DISCUSSION}

None of the alternative trapping devices tested in this study collected as many An. aquasalis mosquitoes as the HLC method. The only deviation from this general pattern was the relatively high number of An. aquasalis collected with the MM-Plus trap on the second day of the study. On this day, the other sampling methods, including HLC, did not show a similar, singular event of a high number of collected Anopheles mosquitoes. An explanation was not found.

The difference between the HLC method and the other three collecting methods, and the lack of difference between the alternative traps indicate that, rather than a difference in trap efficiency, $\mathrm{CO}_{2}$ is not sufficiently attractive trapping bait for An. aquasalis. The insufficiency of $\mathrm{CO}_{2}$ as trap bait may be specific to northern
Paramaribo, where An. aquasalis appear to be more anthropophilic than expected and are guided by more complex host-specific cues than $\mathrm{CO}_{2}$ alone.

If carbon dioxide is not efficient as stand-alone bait for An. aquasalis, what is needed to attract these mosquitoes? If the local population of the species is indeed anthropophilic, human emanations, consisting of a complex mix of body odours (Takken \& Knols 1999), may play an important role. Studies on the role of human odours in attracting anthropophilic malaria vectors are ongoing, with a particular focus on African malarial vectors (Knols et al. 1997, Costantini et al. 1998a, Schmied et al. 2008, Jawara et al. 2009). These studies show that host-specific odours play an important role in vector host-finding behaviour and that odour-baited traps, especially when combined synergistically with $\mathrm{CO}_{2}$, may have strong potential to serve as effective methods for entomological surveillance (Jawara et al. 2009). This is something worth considering for Latin American vectors.

Collecting as many mosquitoes as possible may not always be the purpose of mosquito surveillance. When the purpose of a study is to determine species composition and feeding behaviour over time between indoor, perido-

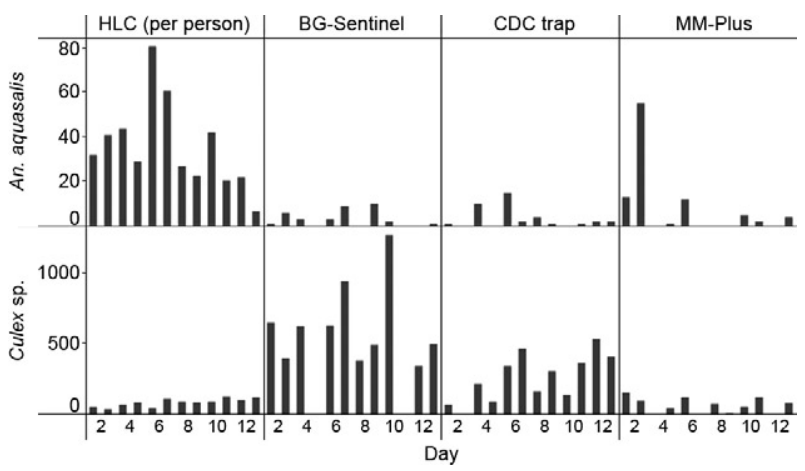

Total number of Anopheles aquasalis and total number of Culex spp per collection method per day. BG-Sentinel: BioGents Sentinel Mosquito Trap; CDC trap: Centers for Disease Control Miniature Light Trap without light; HLC: human landing collection; MM-Plus: Mosquito Magnet ${ }^{\circledR}$ Liberty Plus.

TABLE

Mean number of Anopheles aquasalis and Culex spp mosquitoes per collection method per day

\begin{tabular}{lccc}
\hline & Collection days & $\begin{array}{c}\text { Mean number of } \\
\text { An. aquasalis per day }\end{array}$ & $\begin{array}{c}\text { Mean number of } \\
\text { culicine mosquitoes per day }\end{array}$ \\
\hline HLC (per person) & 12 & $35.58 \pm 19.84^{a}$ & $88.71 \pm 29.17^{a}$ \\
BG-Sentinel & 10 & $3.50 \pm 3.63^{b}$ & $626.40 \pm 287.72^{b}$ \\
CDC trap & 12 & $3.17 \pm 4.63^{b}$ & $263.00 \pm 170.28^{a}$ \\
MM-Plus & 9 & $10.22 \pm 14.48^{b}$ & $88.44 \pm 45.04^{a}$
\end{tabular}

$a, b$ : results with the same letter do not differ significantly ( $\mathrm{p}>0.05)$; BG-Sentinel: BioGents Sentinel Mosquito Trap; CDC trap: Centers for Disease Control Miniature Light Trap without light; HLC: human landing collection; MM-Plus: Mosquito Magnet ${ }^{\mathbb{B}}$ Liberty Plus. 
mestic and outdoor areas, like Rodríguez et al. (2009) did in Colombia, the collection methods used should have a correlation with the HLC method with respect to the relative amount and composition of collected mosquito species over time. None of the tested alternative collection methods in our study showed a correlation with the HLC method with respect to the amount of An. aquasalis mosquitoes collected over time. These data indicate that none of the alternative methods were reliable for determining the malaria vector potential of An. aquasalis.

Analysis of the collected numbers of An. aquasalis per trap found that the MM-Plus trap shows some potential as an alternative for collecting this vector, when the missing data is considered. Studies on propanepowered traps, like the MM-Plus, have demonstrated that they provide relatively good results and are easy to use (Kline 2002). The MM-Plus is generally used with octenol, which is thought to act as a synergist in attracting mosquitoes when combined with carbon dioxide (Takken \& Kline 1989, Kline et al. 1991). Here, we tested the trapping device with only carbon dioxide as bait to allow for fair comparison between traps. However, using MM-Plus with octenol or host-specific attractants may significantly increase the yield of An. aquasalis. The advantage of the MM-Plus over the CDC light trap and the BG-Sentinel trap is its functional longevity. It can function for weeks without needing new batteries or additional propane and may in time even be used as a tool in the integrated mosquito control rather than for mosquito monitoring (Kitau et al. 2009).

The CDC trap and the BG-Sentinel trap showed promising results in previous studies with anophelines (Faye et al. 1992, Davis et al. 1995, Schmied et al. 2008). Using the CDC trap without light decreases the vast amount of insects of no-interest in the collections and may even increase the yield of insects of interest (Carestia \& Savage 1967). Thus, when using the CDC trap with alternative attractants, the light source is often eliminated as bait (Takken \& Kline 1989). In our study, the CDC trap showed potential as a tool for collecting Culex mosquitoes, but failed to be effective in the collection of An. aquasalis.

The advantage of the BG-Sentinel trap is that it is very compact and easy to transport. BG-Sentinel traps are generally provided with BG-Lure, a mosquito attractant containing ammonia, lactic acid and fatty acids that has been developed to mimic human skin odour. Studies with different vector mosquito species show it to be a fairly good trapping method for collecting large numbers of female mosquitoes when compared with the CDC light trap, especially when used in combination with carbon dioxide (Maciel-de-Freitas et al. 2006, Bhalala \& Arias 2009). Using these traps in combination with BG-Lure or other human host-specific baits, such as a worn sock emanating foot odour, may increase its efficiency in collecting anthropophilic An. aquasalis.

In addition to reducing infection risk, the benefits of using collection methods other than HLC include lower costs and the possibility for long-term, more frequent collections. Further, the element of differences in individual attractiveness, an aspect inherent to the HLC method (Khan et al. 1965, Knols et al. 1995), is eliminated. However, use of $\mathrm{CO}_{2}$ as bait has some disadvantages; generally, $\mathrm{CO}_{2}$ is not easy to transport and use in remote areas. The use of propane-powered traps overcomes this disadvantage. Recent findings have demonstrated that sugar-fermenting yeast can serve as a reliable alternative source for $\mathrm{CO}_{2}$ production in mosquito traps (Smallegange et al. 2010). This finding may help solve some logistical problems and allow for a wider use of this attractant in mosquito monitoring tools.

This study indicates that $\mathrm{CO}_{2}$ as a single attractant is not sufficient to trap An. aquasalis. Since the HLC method is very efficient, the An. aquasalis obviously require more host-specific cues for attraction. Knowing what attracts this mosquito species will lead to increased opportunities for the development of alternative collection methods.

\section{ACKNOWLEDGEMENTS}

To Dr Gustavo Bretas (PAHO-Suriname), for his advice and support, to the entomology team of BOG, for their participation, and to Yde Jongema (WUR), for Culex identifications.

\section{REFERENCES}

Bhalala H, Arias JR 2009. The Zumba mosquito trap and BG-Sentinel trap: novel surveillance tools for host-seeking mosquitoes. $J \mathrm{Am}$ Mosq Control Assoc 25: 134-139.

Carestia RR, Savage LB 1967. Effectiveness of carbon dioxide as a mosquito attractant in the CDC miniature light trap. Mosq News 27: 90-92.

Costantini C, Gibson G, Sagnon N, Della Torre A, Brady J, Coluzzi M 1996. Mosquito responses to carbon dioxide in a west African Sudan savanna village. Med Vet Entomol 10: 220-227.

Costantini C, Sagnon NF, Della Torre A, Diallo M, Brady J, Gibson G, Coluzzi M 1998a. Odor-mediated host preferences of West African mosquitoes, with particular reference to malaria vectors. Am J Trop Med Hyg 58: 56-63.

Costantini C, Sagnon NF, Sanogo E, Merzagora L, Coluzzi M 1998b. Relationship to human biting collections and influence of light and bednet in CDC light-trap catches of West African malaria vectors. Bull Entomol Res 88: 503-511.

Davis JR, Hall T, Chee EM, Majala A, Minjas J, Shiff CJ 1995. Comparison of sampling anopheline mosquitoes by light-trap and human-bait collections indoors at Bagamoyo, Tanzania. Med Vet Entomol 9: 249-255.

Deane LM 1986. Malaria vectors in Brazil. Mem Inst Oswaldo Cruz 81 (Suppl. II): 5-14.

Deane LM, Causey OR, Deane MP 1948. Notas sôbre a distribuição e a biologia dos anofelinos das regiões nordestina e amazônica do Brasil. Rev Serv Esp Saude Publica 1: 827-965.

Faye O, Diallo S, Gaye O, Ndir O, Faye O 1992. Comparative efficacy of the use of CDC light traps and humans to sampling Anopheles populations. Results obtained in the area of Bignona (Senegal). Bull Soc Pathol Exot 85: 185-189.

Ferreira E 1964. Distribuição geográfica dos anofelinos no Brasil e sua relação com o estado atual da erradicação da malária. Rev Bras Malariol Doencas Trop 16: 329-348.

Flores-Mendoza C, Cunha RA, Rocha DS, Lourenço-de-Oliveira R 1996. Identification of food sources of Anopheles aquasalis (Diptera: Culicidae) by precipitin test in the state of Rio de Janeiro, Brazil. Rev Saude Publica 30: 129-134. 
Flores-Mendoza C, Lourenço-de-Oliveira R 1996. Bionomics of Anopheles aquasalis Curry 1932, in Guaraí, state of Rio de Janeiro, Southeastern Brazil-I. Seasonal distribution and parity rates. Mem Inst Oswaldo Cruz 91: 265-270.

Gillies MT 1980. The role of carbon dioxide in host-finding by mosquitoes (Diptera: Culicidae): a review. Bull Entomol Res 70: 525-532.

Gillies MT, Wilkes TJ 1969. A comparison of the range of attraction of animal baits and of carbon dioxide for some West African mosquitoes. Bull Entomol Res 59: 441-456.

Healy TP, Copland MJ 1995. Activation of Anopheles gambiae mosquitoes by carbon dioxide and human breath. Med Vet Entomol 9: $331-336$

Jawara M, Smallegange RC, Jeffries D, Nwakanma DC, Awolola TS, Knols BGJ, Takken W, Conway DJ 2009. Optimizing odor-baited trap methods for collecting mosquitoes during the malaria season in the Gambia. PLoS One 4: e8167.

Khan AA, Maibach HI, Strauss WG, Fenley WR 1965. Screening humans for degrees of attractiveness to mosquitoes. J Econ Entomol 58: 694-697.

Kitau JJ, Rwegoshora RR, Rwegoshora D, Matowo J, Mosha FW, Magesa SM 2009. The effect of combined use of Mosquito Magnet Liberty Plus ${ }^{\mathrm{TM}}$ trap and insecticide treated net on human biting rate of Anopheles gambiae s.s. and Culex quinquefasciatus. Tanzan J Health Res 11: 84-89.

Kline DL 2002. Evaluation of various models of propane-powered mosquito traps. $J$ Vector Ecol 27: 1-7.

Kline DL, Allan SA, Bernier UR, Posey KH 2006. Olfactometer and large cage evaluation of a solid phase technology for the controlled production of $\mathrm{CO}_{2} . J$ Am Mosq Control Assoc 22: 378-381.

Kline DL, Wood JR, Cornell JA 1991. Interactive effects of 1-octen-3ol and carbon dioxide on mosquito (Diptera: Culicidae) surveillance and control. J Med Entomol 28: 254-258.

Knols BGJ, de Jong R, Takken W 1995. Differential attractiveness of isolated humans to mosquitoes in Tanzania. Trans $R$ Soc Trop Med Hyg 89: 604-606.

Knols BGJ, Takken W, Cork A, Jong RD 1997. Odour mediated, host seeking behaviour of Anopheles mosquitoes: a new approach. Ann Trop Med Parasitol 91 (Suppl. 1): 117-118.

Laubach HE, Validum L, Bonilla JA, Agar A, Cummings R, Mitchell C, Cuadrado RR, Palmer CJ 2001. Identification of Anopheles aquasalis as a possible vector of malaria in Guyana, South America. West Indian Med J 50: 319-321.

Maciel-de-Freitas R, Eiras AE, Lourenço-de-Oliveira R 2006. Field evaluation of effectiveness of the BG-Sentinel, a new trap for capturing adult Aedes aegypti (Diptera: Culicidae). Mem Inst Oswaldo Cruz 101: 321-325.

Mboera LEG 2005. Sampling techniques for adult Afrotropical malaria vectors and their reliability in the estimation of entomological inoculation rate. Tanzan Health Res Bull 7: 117-124.
Mboera LEG, Knols BGJ, Braks MAH, Takken W 2000. Comparison of carbon dioxide-baited trapping systems for sampling outdoor mosquito populations in Tanzania. Med Vet Entomol 14: 257-263.

Mboera LEG, Takken W 1997. Carbon dioxide chemotropism in mosquitoes (Diptera: Culicidae) and its potential in vector surveillance and management programmes. Rev Med Vet Entomol 85: 355-368.

Mbogo CN, Glass GE, Forster D, Kabiru EW, Githure JI, Ouma JH, Beier JC 1993. Evaluation of light traps for sampling anopheline mosquitoes in Kilifi, Kenya. J Am Mosq Control Assoc 9: 260-263.

Panday RS 1977. Anopheles nuñeztovari and malaria transmission in Surinam. Mosq News 37: 728-737.

Póvoa MM, Conn JE, Schlichting CD, Amaral JC, Segura MN, Da Silva AN, Dos Santos CC, Lacerda RN, De Souza RT, Galiza D, Santa Rosa EP, Wirtz RA 2003. Malaria vectors, epidemiology, and the re-emergence of Anopheles darlingi in Belém, Pará, Brazil. J Med Entomol 40: 379-386.

Reeves WC 1953. Quantitative field studies on a carbon dioxide chemotropism of mosquitoes. Am J Trop Med Hyg 2: 325-331.

Rodríguez M, Pérez L, Caicedo JC, Prieto G, Arroyo JA, Kaur H, Suárez-Mutis M, de La Hoz F, Lines J, Alexander N 2009. Composition and biting activity of Anopheles (Diptera: Culicidae) in the Amazon region of Colombia. J Med Entomol 46: 307-315.

Rozendaal JA 1987. Observations on the biology and behaviour of anophelines in the Surinam rainforest with special reference to Anopheles darlingi Root. Cah ORSTOM ser Ent Med et Parasitol 25: 33-43.

Rozendaal JA 1990. Epidemiology and control of malaria in Suriname: with special reference to Anopheles darlingi, $\mathrm{PhD}$ Thesis, University of Leiden, Netherlands, $171 \mathrm{pp}$.

Rubio-Palis Y 1996. Evaluation of light traps combined with carbon dioxide and 1-octen-3-ol to collect anophelines in Venezuela. J Am Mosq Control Assoc 12: 91-96.

Schmied WH, Takken W, Killeen GF, Knols BGJ, Smallegange RC 2008. Evaluation of two counterflow traps for testing behaviourmediating compounds for the malaria vector Anopheles gambiae s.s. under semi-field conditions in Tanzania. Malar J 7: 230.

Service MW 1993. Mosquito ecology: field sampling methods, 2nd ed., Chapman \& Hall, London, 988 pp.

Smallegange RC, Schmied WH, van Roey KJ, Verhulst NO, Spitzen J, Mukabana WR, Takken W 2010. Sugar-fermenting yeast as an organic source of carbon dioxide to attract the malaria mosquito Anopheles gambiae. Malar J 9: 292.

Takken W, Kline DL 1989. Carbon dioxide and 1-octen-3-ol as mosquito atractants. J Am Mosq Control Assoc 5: 311-316.

Takken W, Knols BGJ 1999. Odor-mediated behaviour of Afrotropical malaria mosquitoes. Annu Rev Entomol 44: 131-157.

Van Thiel PH, Reuter J, Sautet J, Bevere L 1939. On zoophilism and anthropophilism of Anopheles biotypes and species. Riv Malariol 18: 95-124. 